\title{
An Interesting Case Of Concomitant Tuberculosis And Adenocarcinoma Colon
}

\author{
Rashmi Monteiro, Preeti Agrawal*, Shashi Sujanani and K C Vyas
}

Pathology Department, Pacific Medical College and Hospital, Bhilon Ka Bedla, Udaipur, Rajasthan, India

\begin{abstract}
Abdominal tuberculosis and colon carcinomas are commonly seen in patients worldwide. However co-existing gastrointestinal tuberculosis and adenocarcinoma in the same site is unusual and is a rare entity. We here in report an interesting case of tuberculosis and adenocarcinoma occurring concomitantly in the colon, along with metastasis and caseating tuberculosis in mesenteric lymph nodes in a 73-years-old female presenting with pain in abdomen, vomiting and nausea.
\end{abstract}

Keywords: Gastrointestinal Tuberculosis, Mesenteric Lymph Nodes, Colonic Carcinoma

\section{Introduction}

Colonic carcinoma and abdominal tuberculosis are common conditions affecting different parts of the gastrointestinal tract. Tuberculosis is commonly seen affecting the ileocaecal region and carcinoma mostly affects the distal large bowel but simultaneous occurring adenocarcinoma and tuberculosis of colon at the same site is very rare. ${ }^{[1,2]}$ Only 67 cases with concomitant tuberculosis and colonic carcinoma present in the same site are reported in the world literature. ${ }^{[3]}$ Indian literature reports very few cases of carcinoma associated with tuberculosis. ${ }^{[3]}$ We report this case due to its rarity, the diagnostic dilemma encountered and to review the relationship between colon cancer and tuberculosis.

\section{Case Report}

A 73-years-old female of a lower economic strata, housewife, came to Pacific Medical College and Hospital, Udaipur with complaints of pain in abdomen, episodes of vomiting and nausea since 1 month. Pain in abdomen was located more towards the right flank region. The pain was dull aching on and off. She also gave history of loss of weight and appetite. She did not have any complaints of bowel or bladder disturbances. Her past medical and family history showed no contribution to the present illness. On clinical examination, abdomen was soft with a mass palpable in the right flank region which was hard in consistency.

Investigations revealed hemoglobin of $10.0 \mathrm{gms} \%$ with low indices, while TLC, DLC, routine urine examination, serum creatinine, serum sodium, serum potassium and blood sugar were within normal limits. Plain chest x-ray was normal. Ultrasound study of whole abdomen were suggestive of inflammatory /infective etiology. USG guided FNAC from the ileo-caecal mass was suggestive of adenocarcinoma. C.T abdomen \& pelvis showed irregular heterogeneously enhancing thickening involving the ceacum, ileo-caecal junction and appendix with periserosal extention and adjacent peripherally enhancing necrotic lymphnode abutting the ceacal wall. These findings indicated a malignant thickening. Final clinical diagnosis was ileocaecal mass and right hemicolectomy was performed on the patient.

Operative findings revealed a mass in the ileocaecum involving the appendix with multiple enlarged mesenteric lymphnodes. Collection of serous fluid in the cavity was noted. Ileo-caecal resection with ileo-ascending colon end to end anastomosis was done. Post operative period was uneventful. The specimen was sent for histopathological examination.

On gross examination specimen comprised of a part of ileum with caecum \& ascending colon measuring $20 \mathrm{~cm}$. On cutting open a grey-white ulceroproliferative growth was identified at the ileo-caecal junction measuring $3 \mathrm{x}$ $2.5 \times 1.7 \mathrm{~cm}$ which infiltrated upto the serosa. Cut surface of the tumor had a variegated appearance with areas of hemorrhage. The growth was $5 \mathrm{~cm}$ away from the proximal resected margin and $11 \mathrm{~cm}$ away from the distal resected margin. Appendix was not identified in the gross specimen. Total 14 lymphnodes were identified from the mesentry, largest measuring $2 \times 1.5 \times 1 \mathrm{~cm}$ and smallest measuring $0.5 \times 0.5 \times 0.3 \mathrm{~cm}$.

Histopathological examination of the growth showed a tumor composed of cells arranged in glandular pattern. The cells were round to columnar with scanty eosinophilic cytoplasm and hyperchromatic nuclei. Tumor cells were seen infiltrating upto the serosa. Areas adjacent to the 
tumor showed foci of tuberculous infection comprising of epithelioid cell granulomas containing Langhans type of giant cells and mantle of lymphocytes and plasma cells around the granulomas. Sections from 3 lymphnodes showed evidence of metastasis along with tuberculosis



Fig. 1: Cut section shows a grey-white ulceroproliferative growth at the ileo-caecal junction measuring $3 \times 2.5 \times 1.7 \mathrm{~cm}$.

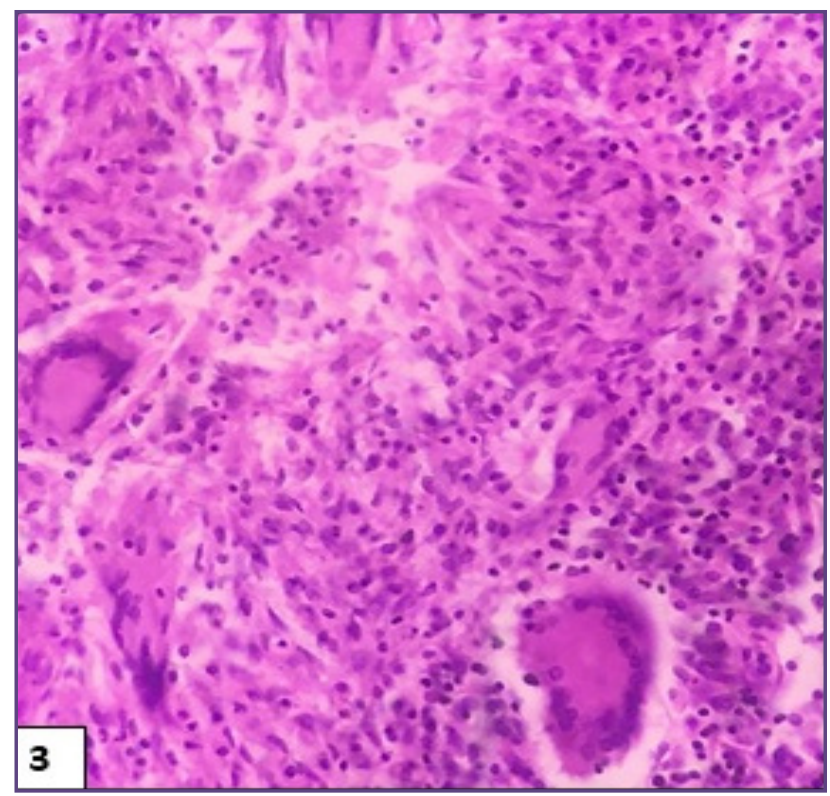

Fig. 3: Epithelioid cells forming granuloma \& Langhans giant cells, H\&E x400. and the remaining 11 lymphnodes showed evidence of caseating tuberculosis.

A diagnosis of coexisting tuberculosis and well differentiated adenocarcinoma caecum was made.

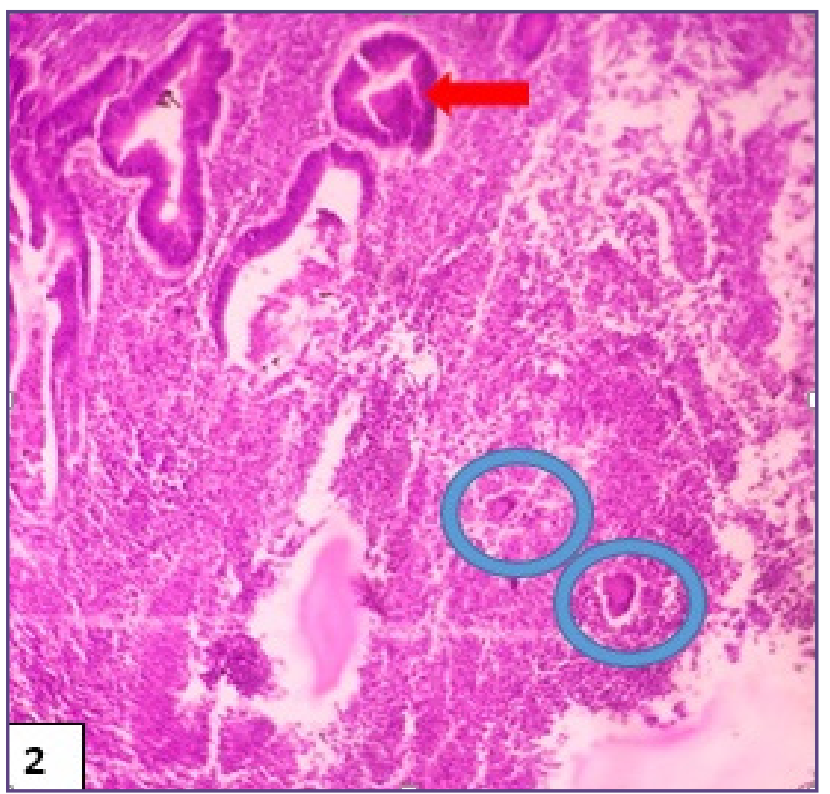

Fig. 2: Red arrow shows tumor arranged in glandular pattern $\&$ tubercular granuloma with Langhans giant cells encircled in blue circles, H\&E x100

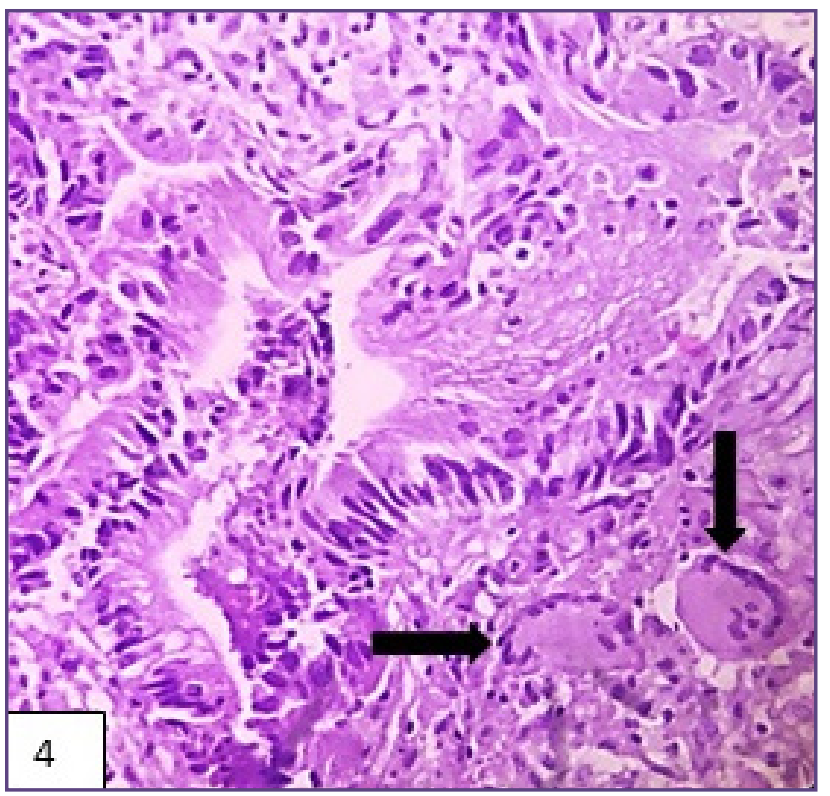

Fig. 4: Tumor arranged in glandular pattern \& black arrows show, Langhans giant cells, H\&E x400. 


\section{Discussion}

The most common site for gastrointestinal adenocarcinoma is the sigmoid colon and for gastrointestinal tuberculosis it's the ileocecal region. ${ }^{[1]}$ Coexisting tuberculosis and carcinoma at the same site is rare and many theories have been proposed regarding its pathogenesis. ${ }^{[1]}$ Few Indian authors suggested that the association of tuberculosis and carcinoma is coincidental..$^{[3]}$ This may be true if the tumor occurs at a site distant from the focus of tuberculosis but both occurring at the same site cannot be mere coincidence. [3] Chronic inflammatory changes seen in mucosa due to diseases like ulcerative colitis, Crohns disease and Schistosomiasis may initiate metaplasia and dysplasia resulting in neoplastic change. ${ }^{[4]}$ Similarly, tuberculosis causes mucosal erosions, ulceration and regenerative changes which may be precursors of carcinoma. ${ }^{[4]}$ Therefore, a proposed theory, that adenocarcinoma arises from the site that is affected by tuberculosis is the most favoured one. ${ }^{[1]}$

Another theory contributing to the concomitant occurrence of carcinoma and tuberculosis, is that factors causing disturbance of host immunity like severe weight loss and malnutrition that are usually seen in advanced carcinomas, leads to increased susceptibility to tuberculosis. ${ }^{\left[{ }^{[3]}\right.}$ Activation and endogenous reinfection of a dormant tubercular lesion may occur due to invasion by carcinoma. Tumor antigens and peptides produced in a carcinoma may also cause the organism of tuberculosis to proliferate by disturbing the milieu of a granuloma.$^{[3]}$ This theory may be true in our case considering the age of the patient, the lower economic conditions and the absence of previous history of active tuberculosis.

Clinical diagnosis of concomitant tuberculosis and cancer can be troublesome to many clinicians due to overlapping symptoms and clinical features. Sometimes microscopic examination of these granulomas may be confusing for a pathologist since they can be confused with sarcoid-like granulomas that occur in response to tumor antigens. ${ }^{[1]}$ Granulomatous reactions are noted in lymphnodes draining carcinoma. Such granulomatous reactions are also noted in the tumor stroma in breast, renal and hepatocellular carcinomas but granulomas within the stroma of colon cancers are rare ${ }^{[1]}$ In addition to this other microscopic features such as caseous necrosis and Langhans-type giant cells is suggestive of tuberculosis.

\section{Conclusion}

The prognosis of carcinoma occurring with tuberculosis at the same site may be favourable since such cases show clinical symptoms at an early stage of the disease. Occurrence of carcinoma and tuberculosis simultaneously in this case highlights the need to rule out their coexistence in patients by clinicians and pathologists.

\section{Acknowledgements}

The authors would like to acknowledge the clinical colleagues and technical staff in the Histopathology laboratory at Pacific Medical College \& Hospital, Udaipur.

\section{Funding}

No funding is involved as this report deals with documentation of a rare case.

\section{Competing Interests}

The authors declare no competing interest.

\section{Reference}

1. Sharma S, Vijay Kumar AK, Kakkar N, Gupta V.Coexisting tuberculosis and adenocarcinoma of colon. Indian J PatholMicrobiol 2016;59:560-1.

2. Ikhwan SM, Bob LS, Zin AA, Zaidi Z. Co existing Abdominal Tuberculosis and Mucinous Adenocarcinoma of Colon: Coincidence or Causal Nexus? IOSR Journal of Dental and Medical Sciences. 2013;5(5):34-38.

3. Chakravartty S, Chattopadhyay G, Ray D, Choudhury CR, Mandal S. Concomitant tuberculosis and carcinoma colon: coincidence or causal nexus?. Saudi journal of gastroenterology: official journal of the Saudi Gastroenterology Association. 2010 Oct;16(4):292.

4. Jairajpuri ZS, Rana S, Jetley S. Coexistent colonic tuberculosis and mucinous adenocarcinoma: A causal or a casual link?. International Journal of Health \& Allied Sciences. 2014 Jul 1;3(3):190.

*Corresponding author:

Dr. Preeti Agrawal, Pacific Medical College and Hospital, Bhilon ka bedla, Pratap Pura, N.H.27, Udaipur, Rajasthan-313001 INDIA

Phone: +91 8854801375

Email: preetibagrawa179@gmail.com

Financial or other Competing Interests: None. 